\title{
Dark fringe interferometry: Multi-beam configuration of the Alcatel nulling interferometer MAII and recent results
}

\author{
Christophe Buisset ${ }^{1,2} \dagger$, Xavier Rejeaunier ${ }^{2}$, \\ Yves Rabbia $^{1}$, Cyril Ruilier ${ }^{2}$, Marc Barillot ${ }^{2}$, and Anders Karlsson ${ }^{3}$ \\ ${ }^{1}$ O.C.A., Dpt GEMINI, UMR CNRS 6203, Av. Copernic, 06130 Grasse, FRANCE \\ email: Christophe.Buisset@obs-azur.fr \\ ${ }^{2}$ Alcatel Alenia Space, Cannes, FRANCE \\ ${ }^{3}$ ESA, Estec, Noordwijk, The NETHERLAND
}

\begin{abstract}
Direct detection and spectroscopic characterization of earth-like exoplanets require to eliminate the starlight in the recorded signal at a rejection level (collected flux / residual flux) around $10^{6}$ in the thermal infrared domain. Another need is to reach angular resolution in the range 0.1 to 0.01 arcsec. In this context, nulling interferometry is recognized as the preferred instrumental approach. In the framework of the ESA-Darwin mission, preliminary studies for nulling systems have been initiated, the prime goal to achieve being mastering nulling process. In this purpose, Alcatel Space has developed a nulling test-bench, operating in near infrared $(1.55 \mu \mathrm{m})$, not only to demonstrate capability of obtaining an appropriate rejection level but also to study and characterize the use of emerging techniques. The Darwin system will ultimately benefit from these results. In this paper, we report the most recent configuration of the Alcatel Space nulling breadboard which uses the multi-beam recombination technique, current baseline for Darwin. The results obtained with this version shows rejection performance at nearly $10^{5}$ (stable level) with a polychromatic source $(\lambda=1.55 \mu \mathrm{m}, \Delta \lambda=80 \mathrm{~nm})$.
\end{abstract}

Keywords. Exoplanets, Direct detection, Nulling interferometry, Multi-beam recombination, ESA-Darwin

\section{Introduction}

In the framework of the DARWIN ESA mission ( direct detection and spectral characterization of earth like exo-planets)(ESA(2000)), Alcatel Alenia Space has developed the test bench MAII which first intended to demonstrate the ability of obtaining high and stable rejection ratio $\rho \approx 10^{6}$. It also serves for testing emerging technologies via ESA contract and acquiring an experience in the field of nulling interferometry so as to develop a cryogenic version of the bench, operating at $\lambda \approx 10 \mu \mathrm{m}$.

The MAII test bench currently operates at $\lambda \approx 1,55 \mu \mathrm{m}$ (telecom spectral domain), fiber and integrated optic technologies being well developed in this wavelength domain. This test bench has already demonstrated the ability of obtaining rejection ratio $\rho \approx 10^{5}$ (monochromatic) and $\rho \approx 10^{4}$ (polychromatic) using an integrated optic combiner (Weber et al. (2004)).

We present a new configuration of the MAII bread board in which the modal filtering and recombination are made using one single-mode fiber. In a first part, we describe the breadboard global architecture. In a second part, we focus on main MAII subsystems

$\dagger$ Present address: Observatoire de la Côte d'Azur, Avenue Copernic, 06130 Grasse, FRANCE 
and their functionality and in a third part, we present the nulling results obtained in February 2005.

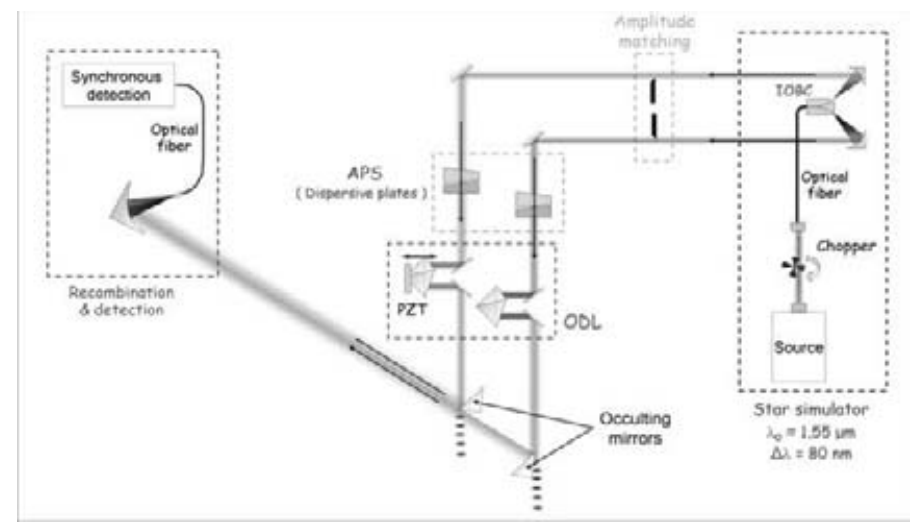

Figure 1. MAII breadboard.

\section{The breadboard}

The MAII breadboard is represented on Figure 1. It consists of a 2-arm interferometer in which an achromatic $\pi$-phase shifting between the fields that will interfere. High contrast interferometry needs for a fully symmetric design Serabyn (2000)), implying that same optical elements must be present along each arm of the interferometer.

The breadboard is composed of three parts : the star simulator, the central part of the interferometer and the recombination and detection part. The central part of the interferometer contains the elements needed for introducing the achromatic $\pi$-phase shifting and matching the amplitude and phase of the fields that will interfere. The polarization matching is achieved by an accurate alignment of the optics what leads to a differential rotation angle of polarization of about 0.1 degree. In the following part, we describe the functionality of each subsystem of the breadboard.

\subsection{Star Simulator, amplitude matching and OPD matching}

The light emitted by the source enters the integrated optic component (IOBC) via a single mode optical fiber. The IOBC delivers two beams collimated by off axis parabolas. These beams stand for the output beams of two telescopes. Because of the birefringent behaviour of the IOBC, only simple polarization measurement can be done.

Knive edges are translated inside the beams to reach the amplitude matching. With the use of high precision translation stage, relative flux difference between the beams is less than $10^{-3}$.

Two identical Optical Delay Lines (ODL) are installed along each arm of the interferometer. These ODL are equiped with a corner cube instead of the classical rooftop. The OPD matching is reached by translating the corner cube along one arm of the interferometer. On the other arm, the similar but static delay line maintains the symmetry of the interferometer. The OPD is manually and real-time adjusted.

\section{2. $\pi$ phase shifting and chromatic correction (APS)}

The Achromatic Phase Shifter (APS) is composed of 2 wedged dispersive plates of different materials installed along each arm of the interferometer. A convinient combination 
of the plates thickness and the use of the ODL yields the achromatic $\pi$ phase shifting between the arms of the interferometer.

\section{3. recombination and detection}

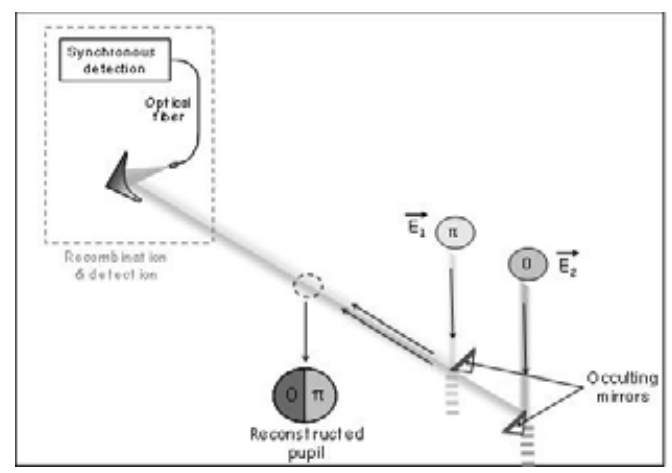

Figure 2. MAII breadboard.

Multibeam mode for recombination consists of:

- Synthesizing a "reconstructed pupil" from 2 half-pupils. Each half-pupil is taken from each arm of the interferometer by occulting mirrors. Consequently, a $\pi$ phase shifting remains between each part of the reconstructed pupil.

_ Recombining and filtering the fields with unique single-mode fiber. An off-axis parabola makes the beams focused at the entry of the fiber.

\section{Results}

In February 2005, we obtained $\rho=6.7 \times 10^{4}$ as the best single polarization nulling performance for a polychromatic source with $5 \%$ of spectral bandwidth. Values higher than $5.10^{4}$ appear on several time intervals of typically 5 seconds.

\section{Conclusion}

We have presented the latest version of the MAII breadboard used for demonstrating the feasability of the multibeam-recombination. The results we obtained demonstrate the feasibility of this kind of recombination for obtaining high rejection ratio, compatible with the ESA-DARWIN performance requirement. To go further, replacement of the IOBC by a non birefringent source and automatic control of the OPD should improve improve the null performance and its stability.

\section{References}

ESA July 2000, Darwin, The infrared Interferometer, ESA-SCI (2000) 12

Weber, et al. 2004, Proc. SPIE Vol. 5491, Astronomical telescopes and instrumentation, p. 842

Serabyn 2000, Proc. SPIE Vol. 4006, Interferometry in Optical Astronomy, p. 328-339 

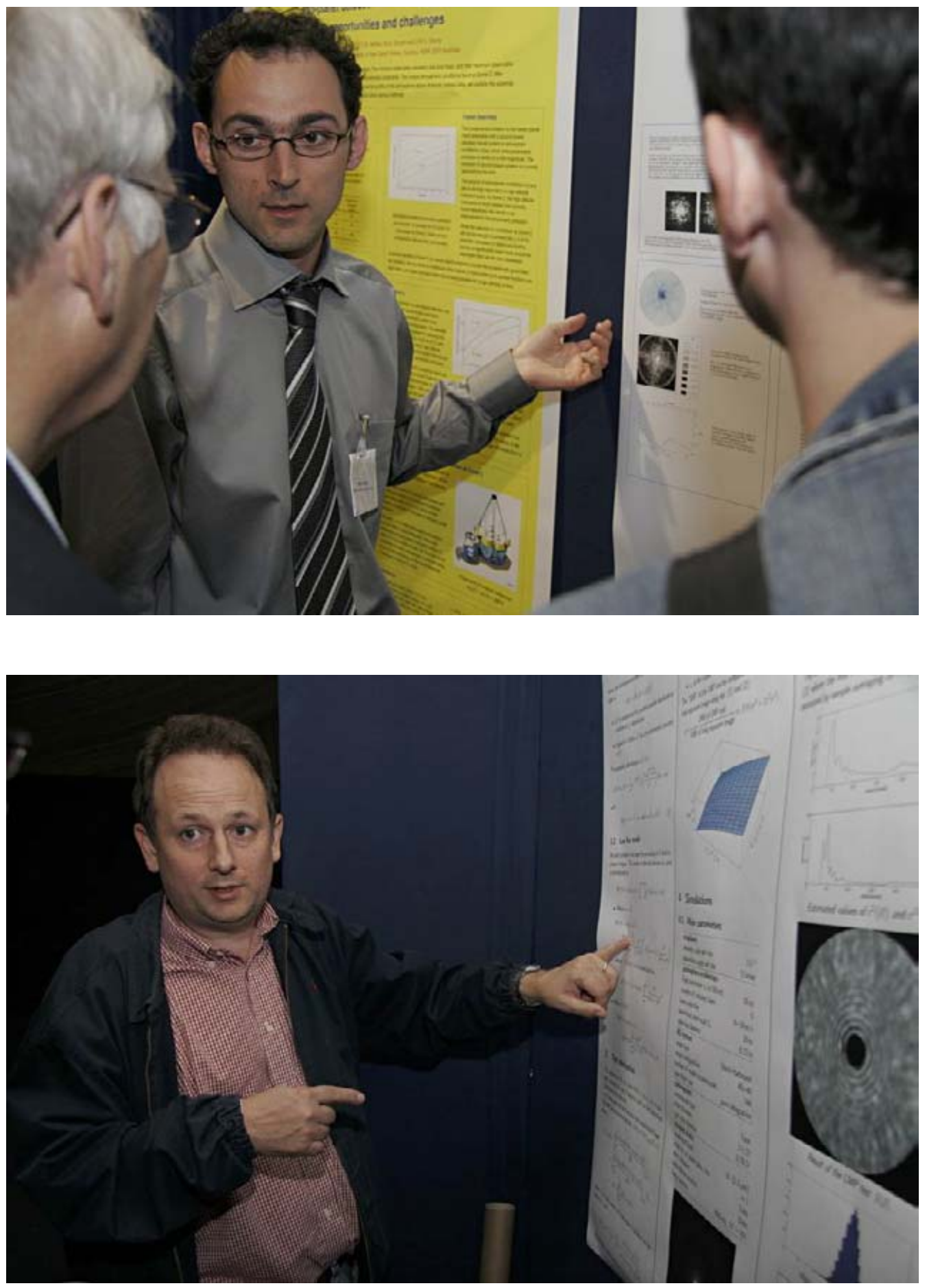

All photographs: Laurent Thareau [1.thareau@free.fr]. 